\title{
Pollution dans les parkings souterrains et risques d'exposition
}

T. vu Duc et C.M.P. Favez

Institut Universitaire de Médecine du Travail et d'Hygiène Industrielle, 1052 Le Mont sur Lausanne

Thème

Les caractéristiques d'un aérosol de gaz d'échappement des véhicules à moteur sont déterminées dans un milieu confiné par opposition aux mesures usuellement effectuées dans l'environnement général. L'avantage réside dans des prélèvements à court terme et en l'absence d'interférences extérieures.

Le niveau de concentration des polluants et la taille des particules déterminent le degré de risque.

\section{Polluants étudiés:}

Poussières, plomb ( $\mathrm{Pb}$ ), cadmium ( $\mathrm{Cd}$ ), manganèse $\left(\mathrm{Mn}_{\mathrm{n}}\right.$ ), hydrocarbures aromatiques polycycliques (HAP), monoxyde de carbone ( $\mathrm{CO}$ ).

\section{Méthodes}

Prélèvements: Poussières collectées sur filtres par échantilionneur de fort volume $\left(40 \mathrm{~m}^{3} / \mathrm{h}\right)$ et impacteur Andersen $\left(20 \mathrm{~m}^{3} / \mathrm{h}\right)$.

Analyses: Les métaux sont analysés par absorption ato mique sans flamme, les HAP par chromatographie liquide haute pression, et le Co par infrarouge non dispersif.

La distribution des particules est faite selon la méthode graphique. Le diamètre moyen correspondant à la masse (MMD) et la déviation standard géométrique $\left(\sigma_{G}\right)$ caracténisent cette distribution considérée comme étant Iog-normal.

\section{Résultats et commentaires}

TABLEAU 1: DISTRIBUTION DES POLLUANTS EN FONCTION DE LA T'AILLE DES PARTICULES

\begin{tabular}{lccccc}
\hline & $\begin{array}{c}\text { MMD } \\
\mu \mathrm{m}\end{array}$ & $\begin{array}{c}\text { *Domaine } \\
\mu \mathrm{m}\end{array}$ & $\sigma_{\mathrm{G}}$ & $\begin{array}{c}<, 1,1 \\
\mu \mathrm{m} \%\end{array}$ & $\begin{array}{c}<3,3 \\
\mu \mathrm{m} \%\end{array}$ \\
\hline Poussières & 3,8 & $1,1-7,0$ & 10,2 & 35 & 53 \\
$\mathrm{~Pb}$ & 0,6 & $0,5-0,8$ & 6,9 & 63 & 81 \\
$\mathrm{Cd}$ & 0,4 & $* *$ & 23 & 64 & 75 \\
$\mathrm{Mn}$ & 5,4 & $2,5-8,4$ & 3,4 & 8 & 29 \\
BaP & 0,06 & $0,02-0,09$ & 71 & 81 & 88 \\
BghiPER & 0,06 & $0,02-0,10$ & 32 & 85 & 91 \\
\hline
\end{tabular}

* Variation des 7 échantililons prélevés w: Analyses cumulées

Le MMD est le diamètre aérodynamique pour lequel $50 \%$ de la masse des particules sont inférieurs ou égaux à la taille mentionnée tandis que $\sigma_{\mathrm{G}}$ définit la dispersion. Excepté le manganèse, les polluants se trouvent incorporés principalement dans les poussières de très petite taille. Le MMD du Pb est semblable à celui de l'environnement général. Il n'est pas affecté par le vieillissement des particules. On l'explique par le fait que les particules de plomb sont émises dans une large gamme de dimension et que les particules de 0,6 $\mu \mathrm{m}$ constituent la taille la plus stable. Ce sont celles-là qui sont captées par les appareils de prélèvement.
TABLEAU 2: CONCENTRATION DES POLLUANTS DANS LE PARKING

\begin{tabular}{lllllll}
\hline $\begin{array}{l}\text { Poussières } \\
\mu g / m^{3}\end{array}$ & $\begin{array}{l}\mathrm{Pb} \\
\mu \mathrm{g} / \mathrm{m}^{3}\end{array}$ & $\begin{array}{l}\mathrm{Cd} \\
\mathrm{ng} / \mathrm{m}^{3}\end{array}$ & $\begin{array}{l}\mathrm{Mn} \\
\mathrm{ng} / \mathrm{m}^{3}\end{array}$ & $\begin{array}{l}\mathrm{BaP} \\
\mathrm{ng} / \mathrm{m}^{3}\end{array}$ & $\begin{array}{l}\text { BghiPER } \\
\mathrm{ng} / \mathrm{m}^{3}\end{array}$ & $\begin{array}{l}\text { CO } \\
\mathrm{PPm}\end{array}$ \\
\hline $480 \pm 200$ & $19 \pm 6$ & $8 \pm 4$ & $120 \pm 30$ & $44 \pm 23$ & $170 \pm 70$ & $94 \pm 30$ \\
MAC $^{* *}: \mathrm{mg}$ & $\mu \mathrm{g}$ & $\mu \mathrm{g}$ & $\mathrm{mg}$ & $\mathrm{ng}$ & & $\mathrm{PPm}$ \\
20 & 100 & 50 & 5 & 150 & & 50 \\
\hline
\end{tabular}

* Moyenne pendant les heures de prélèvement (8-22h). Taux d'occupation du parking pendant la même période: $3500 \pm 700$ voitures.

** Valeurs MAC de divers pays.

Seul le Co dépasse la valeur MAC, mais celle-ci est établie pour une exposition continue de $8 \mathrm{~h}$. Il se pose cependant la question de l'exposition momentanée des usagers du parking et des travailleurs à des pointes transitoires régulièrement enregistrées, de plus de 200 ppm.

TABLEAU 3: COEFFICIENTS DE CORRELATION ENTRE LES DIVERS POLLUANTS

\begin{tabular}{llllll}
\hline Poussières & $\mathrm{Pb}$ & $\mathrm{BaP}$ & BghiPER & $\mathrm{Mn}$ & $\mathrm{CO}$ \\
& 0,90 & 0,85 & 0,64 & 0,87 & 0,87 \\
$\mathrm{~Pb}$ & 0,80 & 0,61 & 0,85 & 0,94 \\
& $\mathrm{BaP}$ & 0,83 & 0,77 & 0,91 \\
& & BghiPER & 0,59 & 0,85 \\
& & & $\mathrm{Mn}$ & 0,71 \\
& & & & $\mathrm{CO}$
\end{tabular}

La meilleure corrélation est obtenue entre le $\mathrm{Pb}$ et le $\mathrm{CO}$. Le contrôle des parkings peut être fait plus simplement par la mesure du monoxyde de carbone.

Summary: POLLUTION IN AN UNDERGROUND CAR PARK AND EXPOSURE RISKS. MOSt of the pollutants emitted by cars are distributed in the respirable size range. Frequent transient values of $\mathrm{co}$ higher than $200 \mathrm{ppm}$ are reconded. It is demonstrated that the control of car pollution can be achieved via co measurements which are easier.

Zusammenfassung: LUFTVERSCHMUTZUNG IN EINEM UNTERIRDISCHEN PARKHAUS UND AUSSETZUNGSRISIKO. Die meis ten der durch Kraftfahrzeuge abgegebenen Schadstoffe sind in der grössenordnung von Partikeln verteilt, die eingeatmet werden können. Spitzenwerte von mehr als 200 ppm würden ebenfalls verzeichnet. Die kontrolle der durch Kraftfahrzeuge verursachten verschmutzung kann am einfachten durch die Kohlenmonoxid Messung durchgeführt werden.

Référence: CHAMBERLAIN A.C., HEARD M.J., LITTLER P. and WIFFEN R.D.: The dispersion of lead from motor exhausts. Phil.Trans.R.Soc.Lond.A. 290, 557 (1979) 